\title{
NOTE ON OVERCONVERGENCE IN SEQUENCES OF ANALYTIC FUNCTIONS
}

\author{
J. L. WALSH AND E. N. NILSON
}

In a recent paper [1] the authors have set forth measures of the degree of convergence of families of analytic functions of best approximation (or of minimum norm) in the sense of least pth powers. It is possible to study overconvergence of certain sequences of these functions by employing the concept of exact harmonic majorant developed by Walsh [2]. It is the purpose of the present note to indicate this relationship and some of its consequences.

Let $R$ be a finite region bounded by a finite sum $C_{1}$ of mutually disjoint Jordan curves. Let $S$ be a closed set interior to $R$, bounded by a finite sum $C_{0}$ of mutually disjoint Jordan curves, but such $C_{0}$ separating no point of $R-S$ from $C_{1}$. Let $\phi(z)$ be the function harmonic in $R-S$, continuous in the closure of $R-S$, equal to zero and unity on $C_{0}$ and $C_{1}$, respectively. Let $C_{\sigma}, 0<\sigma<1$, denote the locus $\phi(z)=\sigma$, while $R_{\sigma}$ is the region consisting of $S$ plus the points of $R-S$ where $\phi(z)<\sigma$. Suppose that $f(z)$ is analytic throughout $R_{\rho}, 0<\rho<1$, but coincides on $S$ with no function analy tic throughout $R_{\rho^{\prime}}$ for any $\rho^{\prime}>\rho$.

In the class of functions $F_{M}(z)$ analytic throughout $R$ and such that the integral mean

$$
\mu_{q}\left(F_{M}, C_{1}\right)=\left\{\frac{1}{\tau} \int_{C_{1}}\left|F_{M}(z)\right|^{q} d \psi\right\}^{1 / q} \leqq M
$$

$\left(\psi(z)\right.$ conjugate to $\phi(z)$ in $\left.R-S, \tau=\int_{C_{1}} d \psi=-\int_{C_{0}} d \psi\right)$, the function $\mathcal{F}_{M}(z)$ is defined as the (or a) function of best approximation to $f(z)$ on $S$ if $\mu_{p}\left(f-\mathcal{F}_{M}, C_{0}\right)$ is least. For certain sequences of values of $M$, including sequences $\left\{M_{n}\right\}$ for which $\log M_{n} / n \rightarrow a, 0<a<\infty$, it was shown [1] that

$$
\limsup _{n \rightarrow \infty} \mu_{t}\left(f-\mathcal{F}_{M_{n}}, C_{\sigma}\right)^{1 / n}=e^{a(\sigma-\rho) /(1-\rho)},
$$

provided $0 \leqq \sigma<\rho$, for all $t, 0<t \leqq \infty$; moreover, for $0<t \leqq \infty$ when $\rho \leqq \sigma<1$ and for $0<t \leqq q$ when $\sigma=1$,

$$
\limsup _{n \rightarrow \infty} \mu_{t}\left(\mathcal{F}_{M_{n}}, C_{\sigma}\right)^{1 / n}=e^{a(\sigma-\rho) /(1-\rho)} .
$$

For $t=\infty$, equations (1) and (2) become $\left(f_{n}(z) \equiv \mathcal{F}_{M_{n}}(z)\right)$

$$
\limsup _{n \rightarrow \infty}\left[\max \left|f(z)-f_{n}(z)\right|, \quad z \text { on } C_{\sigma}\right]^{1 / n}=e^{a(\sigma-\rho) /(1-\rho)},
$$

Received by the editors October 24, 1951. 
$0 \leqq \sigma<\rho$, while

$$
\limsup _{n \rightarrow \infty}\left[\max \left|f_{n}(z)\right|, z \text { on } C_{\sigma}\right]^{1 / n}=e^{a(\sigma-\rho) /(1-\rho)},
$$

$\rho \leqq \sigma<1$. Consequently,

$$
\underset{n \rightarrow \infty}{\limsup }\left[\max \left|f_{n+1}(z)-f_{n}(z)\right|, z \text { on } C_{\sigma}\right]^{1 / n}=e^{a(\sigma-\rho) /(1-\rho)},
$$

$0 \leqq \sigma<1$. (That the left-hand member of (5) is not greater than the right-hand member is immediate from (3) and (4). But unless the equality holds, the sequence $\left\{f_{n}(z)\right\}$ converges uniformly throughout some $\bar{R}_{\rho^{\prime}}, \rho^{\prime}>\rho$, to a function coinciding on $S$ with $f(z)$. This is contrary to the assumption on $f(z)$.)

Equation (5) constitutes a necessary and sufficient condition [2, Corollary 2 of Theorem 4] that the function $V(z)=a(\phi(z)-\rho) /(1-\rho)$ be an exact harmonic majorant of the sequence $\left[f_{n+1}(z)-f_{n}(z)\right]^{1 / n}$ in each $R_{\sigma}, 0 \leqq \sigma<1$, and hence in $R$. Thus the conditions of [2, Theorems 5 and 6] are satisfied. We can conclude, therefore, that if for some sequence $\left\{n_{k}\right\}$ and some $\sigma$ the left-hand member of (3) or (4) is less than the right-hand member, then $\left\{f_{n_{k}}(z)\right\}$ converges throughout some neighborhood of each point of $C_{\rho}$ where $f(z)$ is analytic, and conversely. This result is, of course, much broader than our previous result [1, Theorem 4]. Further, if $\left\{f_{n_{k}}(z)\right\}$ exhibits overconvergence on two disjoint arcs of $C_{\rho}$ having a common end point $\alpha$, then $z=\alpha$ cannot be an isolated singularity of $f(z)$.

This reasoning and these conclusions remain valid if $\left\{f_{n}(z)\right\}$ is no longer a sequence of functions of best approximation, but any sequence of functions, each analytic throughout $R$ with integral mean $\mu_{q}\left(f_{n}, C_{1}\right)$ not greater than $M_{n}\left(\log M_{n} / n \rightarrow a\right)$ and (1) and (2) valid with $t=q$ for $\sigma=0$ and $\sigma=1$, respectively. Analogous remarks can be made for the functions of minimum norm: among the functions $G_{m}(z)$ analytic in $R$ with $\mu_{p}\left(f-G_{m}, C_{0}\right) \leqq m$, the function $G_{m}(z)$ is the (or a) function for which $\mu_{q}\left(G_{m}, C_{1}\right)$ is least. There are many other consequences [2] involving rapidly convergent sequences, zeros of approximating functions, etc., which follow immediately.

\section{REFERENCES}

1. J. L. Walsh and E. N. Nilson, Trans. Amer. Math. Soc. vol. 65 (1949) pp. 239-258.

2. J. L. Walsh, Duke Math. J. vol. 13 (1946) pp. 195-234.

HARVARD UNIVERSITY AND

Trinity College 